\title{
BLOQUEIO DO PLANO ERETOR DA COLUNA
}

\section{ERECTOR SPINAE PLANE BLOCK}

Isis Caroline de Sousa Arruda MD'; Wallace Andrino da Silva, MD, PhD, TSA ${ }^{2}$

1. Médica anestesiologista formada pelo programa de anestesiologia do Hospital Universitário Onofre Lopes.

2. Médico anestesiologista do Hospital Universitário Onofre Lopes, residência médica em anestesiologia e doutorado pela Faculdade de Medicina da Universidade de São Paulo, Título Superior em Anestesiologia (TSA) pela Sociedade Brasileira de Anestesiologia (SBA).

Divisão de Anestesiologia, Hospital Universitário Onofre Lopes, Universidade Federal do Rio Grande do Norte (UFRN). Brasil.

Financiamento: Não.

Conflito de interesse: Não.

Divisão de Anestesiologia, Hopistal Universitário Onofre Lopes - Av. Nilo Peçanha, 620, $3^{\circ}$ subsolo, Petrópolis, Natal/RN CEP:59012-300

Submetido: 01 de março; aceito após revisão: 13 de maio, 2020.

\section{ABSTRACT}

Purpose: A review with the objective of studying spinal block is an interphase analgesic block initially described for the treatment of acute and chronic chest wall neuropathic pain. Methods: Literature review from 2016 to 2019, made using specific terms in the computerized databases. The search was made in May 2019, in the computerized databases PubMed (http://www.pubmed.gov), Cochrane Controlled Trials Register (Central, The Cochrane Library --- http://www.thecochranelibrary.com. br) and Lilacs (http: //lilacs.bvsalud. org). The limits used for bibliographic research were: publications in English or Portuguese from 2016 to 2019. The terms used to obtain the studies were: "Block of the erector plane of the spine", "Chest wall blocks", "Interfascial blocks". Results: Since the initial description in 2016, several applications and changes in the original technique have been published. It consists of an easily performed block, a wide area of analgesia and a low rate of complications, which gives it advantages compared to other chest wall blocks. The mechanism of action, dispersion patterns and effective doses of local anesthetics, however, are not yet fully understood.

Key words: pain; anesthesia; erector spinae plane block. Nerve Block

\section{RESUMO}

Objetivo: Revisão com o objetivo de estudar o bloqueio do plano eretor da coluna é um bloqueio analgésico interfascial inicialmente descrito para tratamento de dor neuropática aguda e crônica da parede torácica. Métodos: Revisão da literatura de 2016 a 2019, feita por meio de termos específicos nos bancos de dados informatizados. A 
busca foi feita em maio de 2019, nas bases de dados computadorizadas PubMed (http://www.pubmed.gov), Cochrane Controlled Trials Register (Central, The Cochrane Library - http://www.thecochranelibrary.com.br) e Lilacs (http://lilacs.bvsalud. org). Os limites usados para pesquisa bibliográfica foram: publicações em inglês ou português de 2016 a 2019. Os termos usados para a obtenção dos estudos foram: "Bloqueio do plano eretor da espinha", "Bloqueios da parede torácica", "Bloqueios interfasciais". Resultados: Desde a descrição inicial em 2016, diversas aplicações e modificações na técnica original têm sido publicadas. Consiste em um bloqueio de fácil realização, ampla área de analgesia e baixo índice de complicações, o que lhe atribui vantagens frente a outros bloqueios da parede torácica. O mecanismo de ação, os padrões de dispersão e as doses efetivas de anestésicos locais, entretanto, ainda não estão totalmente esclarecidos.

Descritores: Dor. Anestesia. Bloqueio do plano eretor da espinha. Bloqueio nervoso

\section{INTRODUÇÃO}

O bloqueio do plano eretor da coluna (BPEC) é uma técnica de anestesia regional em que o anestésico local ( $A L)$ é injetado entre o músculo eretor da coluna e processo transverso por agulhamento sob orientação ultrassonográfica, bloqueando ramos dorsais e ventrais dos ramos torácico e abdominal dos nervos espinhais. ${ }^{2}$

A primeira publicação é de 2016 por Forero et al em que 20 mililitros de AL foram injetados a nível da quinta vértebra torácica (T5) para tratamento de dor neuropática grave da parede torácica. Em ambos os casos descritos, o bloqueio foi bem sucedido, produzindo alívio significativo da dor e extenso bloqueio sensorial da região afetada. ${ }^{1}$

O BPEC tem sido aplicado com sucesso em cirurgias diversas, como videotoracoscopia, lobectomia pulmonar, fraturas de costela, mastectomia, biópsia de linfonodo sentinela axilar, cirurgia abdominal, manejo da dor em grandes queimados e em casos de dor neuropática na região torácica ${ }^{3}$, com redução de até $65 \%$ do consumo de opioides no transoperatório ${ }^{19}$.

Outros bloqueios fasciais foram descritos e ganharam espaço devido à simplicidade das técnicas e ao pequeno índice de complicações. Destacamos neste grupo o bloqueio do plano transverso do abdome, o bloqueio da bainha dos retos, o bloqueio do quadrado lombar, os bloqueios peitorais (PEC 1 e 2) e do serrátil.

\section{MÉTODOS}

Revisão da literatura de 2016 a 2019, feita por meio de termos específicos nos bancos de dados informatizados. A busca foi feita em maio de 2019 , nas bases de dados computadorizadas PubMed (http://www.pubmed.gov), Cochrane Controlled Trials Register (Central, The Cochrane Library - http://www.thecochranelibrary.com.br) e Lilacs (http://lilacs.bvsalud. org). Os limites usados para pesquisa bibliográfica foram: publicações em inglês ou português de 2016 a 2019. Os termos usados para a obtenção 
dos estudos foram: "Bloqueio do plano eretor da espinha", "Bloqueios da parede torácica", "Bloqueios interfasciais". Foram aceitos artigos que respondessem à questão norteadora estabelecida e obedecessem aos seguintes critérios de inclusão: que versassem sobre os efeitos, as características clínicas, a eficácia e a segurança dos bloqueios em questão, revisões literárias e pesquisas experimentais realizadas em cadáveres; e que os resumos estivessem disponíveis on-line. Foram obtidos um total de 55 artigos, lidos e analisados integralmente, e destes 16 foram destacados por relevância afinidade com o tema de interesse.

\section{ANATOMIA}

Estudos realizados em cadáveres frescos com injeção de contraste demonstraram que os prováveis locais de ação do BPEC são os ramos dos nervos dorsais e ventrais da coluna torácica ${ }^{5}$. Cada nervo espinhal torácico superior se divide em um ramo dorsal e ventral na sua saída do forame intervertebral. O ramo dorsal passa posteriormente através do forame costotransverso e sobe para o músculo eretor da espinha, onde se divide em ramos lateral e medial. O ramo medial continua ascendendo através dos músculos romboide e trapézio, antes de terminar em ramo cutâneo posterior. O ramo ventral, por sua vez, passa lateralmente, assim como o nervo intercostal, correndo primeiro para o interior da membrana intercostal e, em seguida, no plano entre os músculos intercostais internos. O nervo intercostal termina em um ramo cutâneo anterior, que inerva a parede torácica anterior e a parte superior do abdome. O ramo cutâneo lateral surge do nervo intercostal, perto do ângulo da costela, e sobe superficialmente, emergindo perto da linha axilar média, subdividindo-se em ramos anterior e posterior que suprem a parede torácica lateral. Além desses ramos principais, cada nervo intercostal também dá origem a múltiplos ramos musculares, inervando os músculos intercostais ${ }^{1}$.

O AL deve ser depositado em estreita proximidade do forame costotransverso, ou seja, o mais próximo possível da linha média neuroaxial, de onde ambos os ramos, dorsal e ventral, dos nervos espinhais torácicos são originários. A injeção nessa localização aumenta a probabilidade do AL penetrar no espaço paravertebral e promover analgesia visceral. Com injeções mais laterais, os músculos intercostais internos e externos podem representar barreiras a disseminação do AL.

A técnica original descreve o agulhamento em plano, no sentido céfalo-caudal, com o transdutor linear de alta frequência em plano parassagital a aproximadamente três centímetros da linha média neuroaxial, a nível de T4-T5, onde a injeção de AL foi realizada inicialmente no plano interfascial entre o músculo romboide e o músculo eretor da coluna vertebral e, subsequentemente, profunda ao plano do músculo eretor da espinha ${ }^{3}$. Desde então, a experiência clinica e os estudos cadavéricos indicam que o plano ideal para injeção é profundamente ao plano do músculo eretor da coluna, 
banhando os ramos dorsal e ventral dos nervos espinhais, e nível variável de acordo com área de analgesia pretendida.

Três músculos deverão ser transpostos até atingir a ponta do processo transverso, são eles: o trapézio (superior), romboides principais (meio) e eretor da coluna (inferior). A provável base anatômica para a efetividade do bloqueio é o depósito do $A L$ sob a bainha do músculo eretor. Esta bainha, que individualiza o músculo eretor da coluna dos demais músculos da parede torácica e abdominal posterior possui lacunas anatômicas que, possivelmente, permitem o espalhamento do AL até o espaço paravertebral.

O músculo eretor da coluna, por sua vez, trata-se de um conjunto de três ramos: iliocostal, longuíssimo do dorso e espinhal, que surgem e se inserem em vários componentes ósseos da coluna vertebral, por exemplo: do processo espinhoso ao processo espinhoso, de costela para costela e de processo transversal para processo transversal. Fazem parte dos músculos intrínsecos do dorso e tem a função de manutenção da postura e de alguns movimentos da coluna vertebral.

Segundo Hamilton e Manickam, uma das estruturas anatômicas mais importantes para a adequada disseminação do AL no sentido cefalocaudal é a fáscia toracolombar, que se estende ao longo de todo o tórax e abdome posterior, e se continua com a fáscia nucal no pescoço e consiste de três camadas de fáscias e aponeurose de vários músculos - o grande dorsal, serrátil posterior inferior, oblíquos internos e abdominais transversos. A camada posterior da fáscia insere-se nos processos espinhosos e cobre os músculos da coluna. A camada média é posterior ao quadrado lombar e insere-se nas pontas dos processos transversos e ligamentos intertransversos. As camadas média e posterior envolvem os músculos eretores da coluna. A camada anterior, por sua vez, é uma lâmina anterior ao quadrado lombar e insere-se na face anterior dos processos transversos e ligamentos intertransversos ${ }^{4}$.

\section{TÉCNICA}

Segundo descrição de Luftig et al., inicialmente deve-se expor a região posterior do tórax, posicionando o paciente deitado em decúbito lateral, ou inclinado para frente em posição sentada. ${ }^{9}$ Para a posição em decúbito lateral (com o paciente deitado no lado não afetado), deve-se sentar ao lado da cama de frente para as costas do paciente com o aparelho de ultrassonografia no lado oposto da cama (anterior ao paciente). Para a posição sentada, o paciente deve sentar na beira da cama, inclinado para a frente em uma posição similar à posição sentada da punção lombar, e o examinador deve se posicionar atrás do paciente com o sistema de ultrassonografia localizado no lado oposto do leito (anterior ao paciente). Para todas as posições, levante a cama até um nível em que a agulha, o transdutor e a tela do aparelho de ultrassonografia estejam alinhados de forma que possam ser visualizados com movimentos mínimos de cabeça. 
O nível da punção deve ser definido de acordo com a área que se deseja anestesiar. Recomenda-se que a punção seja realizada aproximadamente no ponto médio dessa área, numa perspectiva crânio-caudal.

O transdutor é inicialmente posicionado na linha média, sobre o processo espinhoso, e deslocado em média três centímetros lateralmente, em busca de estruturas ósseas chave como o processo transverso (PT), a junção costotransversa e a costela, que podem ser diferenciadas por suas formas e profundidades relativas, conforme o transdutor é movido lateralmente a partir da linha média.

Para confirmar a identificação do PT, devemos deslizar a sonda para além do alvo lateralmente, passando pelo ponto de articulação com a costela. A costela posterior adjacente à junção costotransversa é tanto lateral quanto profunda ao PT. Deslizando o transdutor para frente e para trás sobre a junção, a diferenciação entre o PT e costela será clara. O PT será mais superficial, mais reto e mais largo, enquanto a costela será mais profunda, mais arrendondada e mais fina. Após identificado o PT, estabilizar o transdutor e proceder agulhamento alinhado com o eixo longo do feixe de ultrassom a aproximadamente $1-2 \mathrm{~cm}$ de distância.

O local de inserção pode ser cefálico ou caudal à sonda, uma vez que o PT pode ser alcançado de qualquer direção. Cornish et al, porém, enfatizam que o agulhamento deve ser realizado em direção cefálica-caudal, pois assim evitaríamos o ligamento costotransverso anterior ${ }^{10}$.

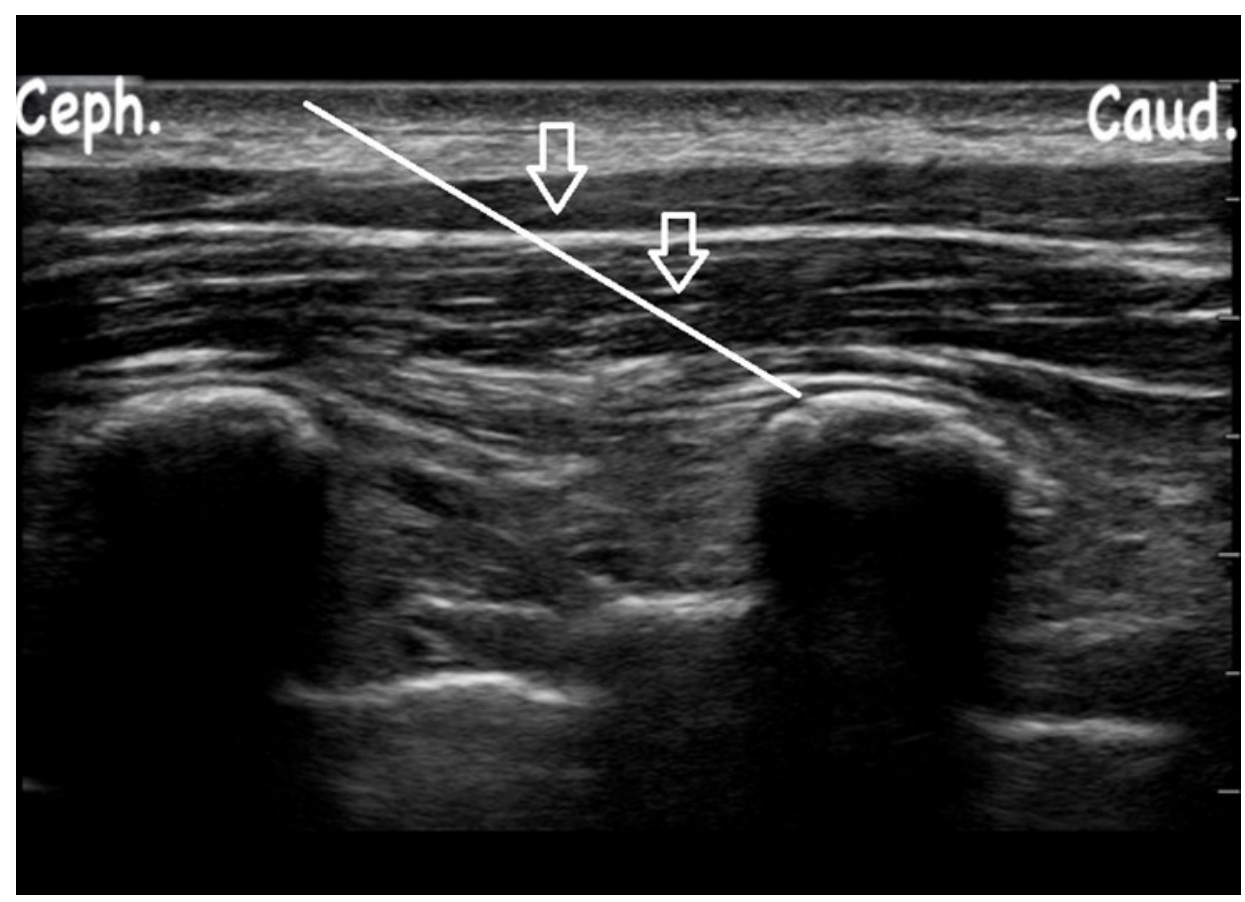

FONTE: AnSO app (Anaesthesia Sonoanatomy) em Head, Neck and Trunk Nerves

Figura 1: Sentido do agulhamento 


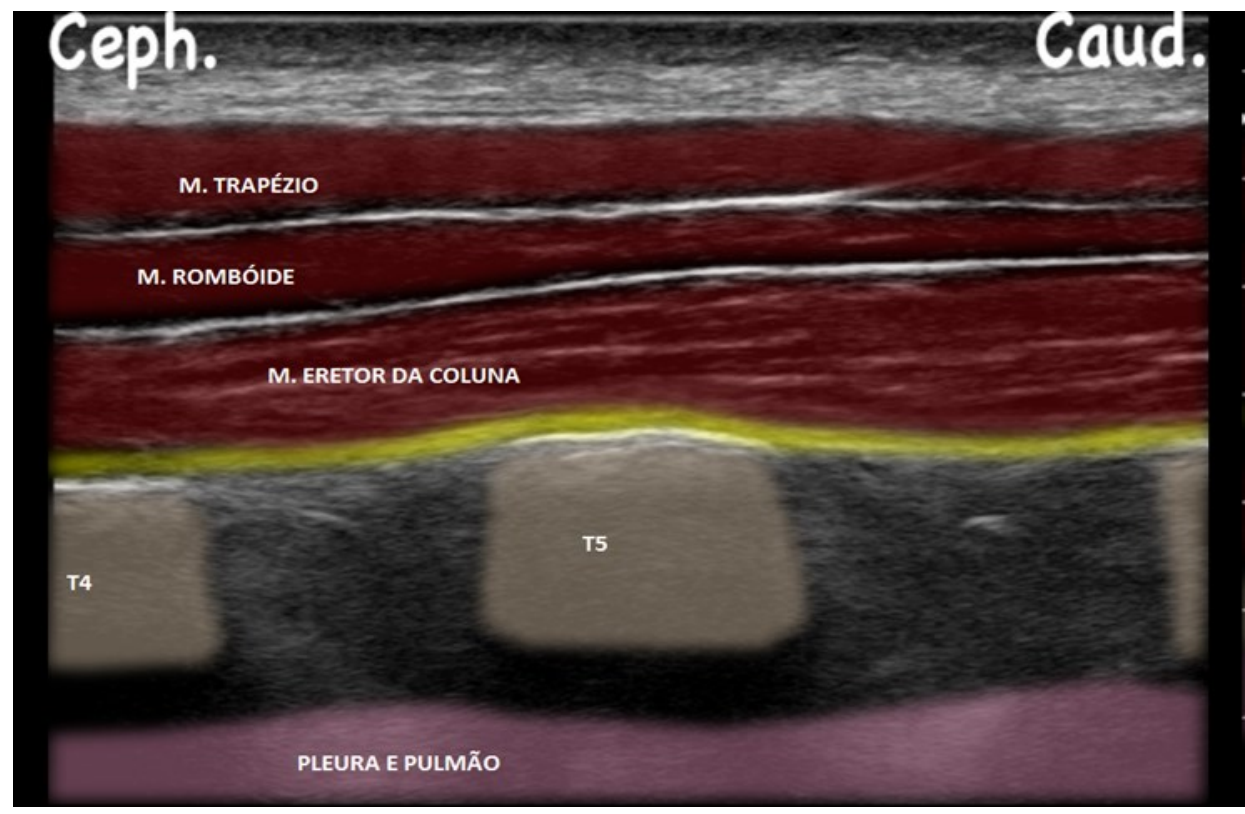

FONTE: AnSO app (Anaesthesia Sonoanatomy) em Head, Neck and Trunk Nerves

Figura 2: Estruturas anatômicas no trajeto da agulha

Após a assepsia do sítio de punção, realizar botão anestésico com lidocaína a 1\% ou a $2 \%$ (caso o paciente não esteja sob anestesia geral) e inserir a agulha de bloqueio (raquianestesia 22G ou $25 \mathrm{G}$ ou agulha epidural Tuohy ou agulha de Stimuplex ${ }^{\circledR}$ ) através da pápula da pele e avançar a agulha em um ângulo de 30 a 45으 em relação à pele em direção ao feixe de ultrassom.

Após a inserção inicial, fazer pequenos ajustes de transdutor e agulha (mantendo o PT à vista) até que a ponta da agulha esteja visível. É possível também realizar mínimos bolus de AL ou soro fisiológico para localização por hidrodissecção.

Com o avançar da agulha, o operador pode sentir "cliques" correspondentes à perfuração das fáscias do trapézio e do rombóide. Quando a ponta finalmente atingir um ponto final firme (periósteo do PT), a injeção deve ser realizada, e a imagem a ser visualizada deve ser de um fluido anecóico separando o músculo eretor da espinha do PT.

Chin et al, enfatizam que a injeção intramuscular deve ser evitada, e a imagem a ser perseguida é a de AL espalhando-se linearmente entre duas camadas hiperecóicas distintas, em vez de puramente entre o músculo hipoecóico e a camada hiperecóica abaixo dele, pois acreditam que assim a penetração no espaço paravertebral é maximizada. Eles recomendam que o processo transverso deve ser usado como backstop para facilidade e segurança do bloqueio.

A maioria dos trabalhos descreve a administração de um volume de $20 \mathrm{ml}$ de $\mathrm{AL}$ em cada hemitórax, obtendo-se, em geral, anestesia do tronco dentro de 30 minutos, com os pacientes referindo parestesias das regiões torácicas anterior, lateral e posterior.

A infusão deve ser realizada em pequenas alíquotas, alternando-se com aspirações, a fim de se evitar injeção intravascular inadvertida. 


\section{MECANISMO DE AÇÃO}

Uma das explicações para o mecanismo de ação desse bloqueio é a dispersão do AL para o espaço paravertebral através de lacunas anatômicas específicas, como o forame costotransverso (espaço entre o ligamento transverso superior e o colo da costela para a passagem do ramo intercostal do nervo espinhal) e as lacunas no ligamento costotransverso (limite posterior do espaço paravertebral). Devido a esse padrão de dispersão, o BPEC é considerado por alguns autores como um bloqueio paravertebral indireto.

Estudos realizados em cadáveres por Forero et al, entretanto, não conseguiram reproduzir esse padrão de dispersão, o que pode ser explicado por variações anatômicas e técnicas, e nos faz considerar que a propagação para o espaço vertebral ocorre mais como exceção do que como regra nesse bloqueio.
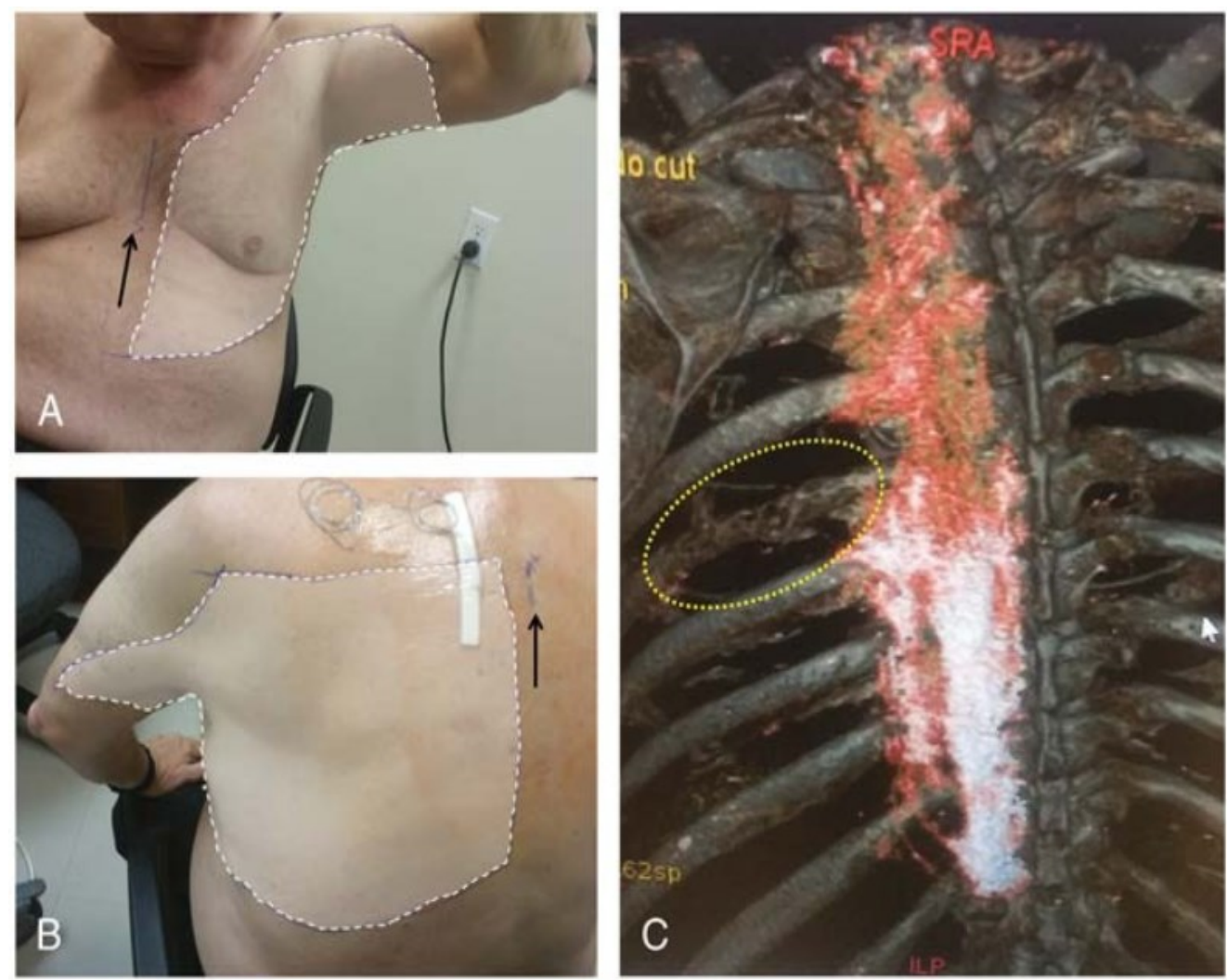

FONTE: Forero, Adhikary, Lopez e Chin (2016 - p.622)

Figura 3: Extensão da perda sensorial cutânea sobre o tórax anterior $(A)$ e posterior (B); C, reconstrução tridimensional da TC demonstrando a extensa dispersão cefalocaudal após injeção de $20 \mathrm{ml}$ de solução de $A L$ em paciente com fratura de costela.

Estudos realizados em um cadáver por Chin et al. com injeção de $20 \mathrm{ml}$ de solução contrastada bilateralmente em T7 mostraram disseminação de AL de T2 a L3 à direita e de C5 a L2 à esquerda. As diferenças de propagação mostram que, havendo variabilidade entre os dois lados de um mesmo indivíduo, é provável que haja 
variabilidade interindividual na disseminação física de anestésico local e consequente intensidade e extensão da analgesia ${ }^{6}$.

Em geral, admite-se disseminação que o AL se estenda por pelo menos três níveis vertebrais cranialmente e quatro níveis caudalmente a partir do local da injeção (para um volume de $20 \mathrm{ml}$ de AL). Investigações adicionais são necessárias para determinar se existe, assim como na peridural, uma relação proporcional entre o volume injetado e a extensão da analgesia.

Para injeções em nível lombar, a premissa acima não é válida, dado que são os limites bem definidos dos espaços paravertebrais em nível torácico, que fazem com que a propagação atinja ambos os ramos ventral e dorsal em vários níveis. Esse padrão estrutural não está presente a nível lombar, o qual não tem limites claros, levando a um espalhamento de AL parcial e impreciso. Não obstante, muitos autores têm obtido analgesia efetiva com injeções a nível lombar para cirurgias abdominais baixas e pélvicas, como confecção ou fechamento de ileostomias, artroplastia total de quadril e cirurgias de fêmur proximal ${ }^{8,11,18}$.

\section{DROGAS UTILIZADAS}

Anestésicos locais são bases fracas que inibem reversivelmente a transmissão nervosa pela ligação aos canais de sódio voltagem dependentes na membrana plasmática do nervo. Todos os AL usados clinicamente compartilham determinadas características estruturais: consistem em um anel aromático lipofílico e uma amina terciária hidrofílica, que se unem entre si por uma ligação éster ou amida. Esta ligação classifica os AL em dois grupos: aminoamidas e aminoesteres. Em nossa prática, as aminoamidas são mais comumente utilizadas, e incluem a lidocaína, a ropivacaína e a bupivacaína. A lidocaína é classificada como medicação de duração intermediária (em média 30-60 minutos), e a ropivacína e bupivacaína de longa duração (em média 120240 minutos).

Para realização do BPEC, qualquer um dos AL citados acima pode ser utilizado. A escolha depende, em grande parte, da duração de ação desejada. O uso de anestésico com vasopressor é uma alternativa para o prolongamento da duração (principalmente da lidocaína, com incremento de até $100 \%$ do tempo de ação), bem como para redução da potencial toxicidade dessas drogas.

Em geral, utilizam-se maiores volumes com baixas concentrações, para que se obtenha uma maior extensão da área de anestesia, respeitando-se a dose recomendada. Chin et al descrevem procedimentos com o uso de ropivacaína a 0,375\%; Tulgar et al realizaram estudos com levobupivacaína a $0,25 \%$. Assim, concluimos que, respeitando o limite de dose tóxica recomendada pelo peso do paciente, é possível utilizar AL em diversas diluições. 
Dentre os aditivos utilizados para incremento da atividade dos $A L$, destacamos a clonidina, um $\alpha_{2}$-agonista de ação direta, que também possui efeitos diretos na condução neural e cujo uso em bloqueios nervosos periféricos prolonga a duração do bloqueio. Entretanto, efeitos colaterais como bradicardia e hipotensão ortostática tem limitado o seu uso. Apesar do ser amplamente difundido, não encontramos estudos na literatura que embasem seu uso especificamente para o BPEC.

\section{VANTAGENS E LIMITAÇÕES}

Uma das vantagens mais significativas do BPEC é, sem dúvidas, a sua simplicidade e segurança. A sonoanatomia é facilmente reconhecível, sendo o PT é um marco ultrassonográfico muito conveniente, pois funciona como backstop para o avanço da agulha, o que contribui para facilidade e redução dos riscos.

Se comparados a outros bloqueios da parede torácica, como o paravertebral, a peridural e o intercostal, por exemplo, o índice e a gravidade das possíveis complicações são muito menores.

Desde os primeiros relatos em 2016 até hoje, só há publicação de um caso de complicação, em que uma paciente candidata a mastectomia desenvolveu uma neuropatia autonômica parcial (síndrome de arlequim), após submetida a BPEC. O processo patológico subjacente envolve a interrupção ipsilateral da via autonômica entre o hipotálamo, coluna intermediolateral da medula espinhal, gânglios simpáticos cervicais e fibras simpáticas pós-ganglionares, resultando em palidez facial ipsilateral relativa e anidrose com supercompensação contralateral. A paciente teve resolução completa dos sintomas após 12 horas da instalação do quadro. ${ }^{14}$

A técnica também se presta bem para inserção de um cateter de demora, que pode ser usado para estender a duração da analgesia conforme necessário. Embora o regime de dosagem ideal exija maiores investigações, o uso de cateter é benéfico em situações onde é esperada dor moderada a grave por mais de 12 horas. Em regimes de manutenção de 2 a 3 dias, houve diminuição no consumo de opioides, com consequente dimunuição de NVPO, e dimunuição da incidência de dor crônica secundária ao procedimento cirúrgico. ${ }^{19,20}$

Poderá ser realizado em pacientes recebendo tratamento com anticoagulantes por se tratar de bloqueio superficial e sem estruturas nervosas e/ou vasculares nobres no trajeto da agulha.

Por sua ampla cobertura analgésica se aplica a várias condições, incluindo controle de dor aguda e crônica das paredes torácica, abdominal e até mesmo da pelve: cirurgias pulmonares, cardíacas, de mama, e pequenos procedimentos de parede torácica como retirada de lipomas, confecção e fechamento de ileostomia, laparotomias, artroplastia de quadril, prostatectomias, cirurgias de fêmur proximal e manejo da dor em queimaduras extensas. ${ }^{11,12,21}$ 
Por outro lado, as desvantagens do BPEC devem ser lembradas. A necessidade de aparelho de ultrassom para sua realização é um fator limitante em muitos serviços, além disso, o operador deve estar familiarizado com a técnica e nunca avançar inadvertidamente a agulha, a menos que a ponta seja claramente visualizada, a fim de evitar punções acidentais de pleura ou medula. A variabilidade interindividual no espalhamento de AL pode resultar em regiões de anestesia menores ou maiores que o esperado. Pacientes com tecido extenso entre a superfície da pele e o PT são candidatos ruins para este bloqueio pela maior dificuldade técnica.

Embora incomum, intoxicação por AL é uma complicação que deve ser considerada em todos os bloqueios, principalmente se as precauções relativas a doses tóxicas e injeções intravasculares forem ignoradas. Outras limitações são anestesia unilateral, o que requer duas punções para anestesia de toda a parede torácica, e por curto período de tempo.

\section{CONCLUSÕES}

O ponto positivo mais significativo do BPEC é, sem dúvidas, sua simplicidade de execução e segurança. A literatura tem mostrado sua vasta aplicabilidade e reduzido índice de complicações quando comparados a outros bloqueios da parede torácica.

Por se tratar de um bloqueio relativamente novo, as evidências atualmente são limitadas para a utilidade clínica, mas acreditamos que por sua fácil reprodutibilidade, pela possiblidade de prolongar seu efeito com uso de drogas adjuvantes e cateteres de longa permanência, e pela vasta área de anestesia obtida, o BPEC irá se consagrar como um bloqueio de grande valor na prática anestésica e na algologia.

\section{REFERÊNCIAS}

1. Forero M, Adhikary SD, Lopez H, Tsui C, Chin KJ. the erector spinae plane block: A novel analgesic technique in thoracic neuropathic pain. Reg Anesth Pain Med. 2016;41(5):621-7.

2. López MB, Cadórniga AG, González JML, Suárez ED, Carballo CL, Sobrino FP. Erector Spinae Block. A narrative review. Central Eur J Clin Res. 2018;1(1):28-39

3. Hamilton DL, Manickam B. Erector Spinae Plane Block for Pain Relief in Rib Fractures. Br J Anaesth. 2017;118(3):474-5.

4. Moore, Keith L. Anatomia Orientada para a Prática Clínica. 4ed. Rio de Janeiro: Guanabara Koogan, 2001.

5. Ivanusic J, Konishi Y, Barrington MJ. A Cadaveric study investigating the mechanism of action of erector spinae blockade. Reg. Anesth Pain Med. 2018;43(6):567-71.

6. Chin KJ, Adhikary S, Sarwani N, Forero M. The analgesic efficacy of pre-operative bilateral erector spinae plane (esp) blocks in patients having ventral hernia repair. Anaesthesia. 2017;7(4):452-60.

7. Hamilton DL, Manickam BP. Is the erector spinae plane (esp) block a sheath block? Anaesthesia. 2017;72(7):915-6. 
8. Tulgar S, Selvi O, Senturk O, Ermis MN, Cubuk R, Ozer Z. Clinical experiences of ultrasound-guided lumbar erector spinae plane block for hip joint and proximal femur surgeries. J Clin Anesth. 2018;47:5-6.

9. Luftig J, Mantuani D, Herring AA, Dixon B, Clattenburg E, Nagdev A. Successful emergency pain control for posterior rib fractures with ultrasound-guided erector spinae plane block. Am J Emerg Med;36(8):1391-6.

10. Cornish PB. Erector Spinae Plane Block: The "Happily Accidental" paravertebral block. Reg Anesth Pain Med. 2018;43(6):644-645.

11. Tulgar S, Thomas DT, Deveci U. Erector spinae plane block provides sufficient surgical anesthesia for ileostomy closure in a high-risk patient. J Clin Anesth 2018;48:2-3.

12. Ueshima $H$, Otake $H$. continuous erector spinae plane block for pain management of an extensive burn. Am J Emerg Med. 2018;36(11):2130.e1-2130.e2.

13. Ahmet MY, Ali A, Erkan CC, Aysenur A, Atila O. Bloqueio do plano do eretor da espinha para analgesia pós-operatória de cirurgia de fratura de múltiplas costelas: relato de caso. Rev Bras Anestesiol 2019;69(1):91-4.

14. Sullivan TR, Kanda P; Gagne S, Costache I. Harlequin syndrome associated with erector spinae plane block. Anesthesiology 2019;131:665.

15. Schwartzmann A, Peng P, Maciel MA, Forero M. Mechanism of the erector spinae plane block: insights from a magnetic resonance imaging study. Can J Anesth Cand'anesthésie 2018;65(10):1165-6.

16. Chin KJ, Malhas L, Perlas A. The Erector spinae plane block provides visceral abdominal analgesia in bariatric surgery: a report of 3 cases. Reg Anesth Pain Med 2017;42(3):372-6.

17. Serkan T, Onur S, Ahiskalioglu A, Ozer Z. Can unilateral erector spinae plane block result in bilateral sensory blockade? Can J Anaesth. 2019;66(8):1001-2.

18. Tulgar S, Senturk O. Ultrasound guided Erector Spinae Plane block at L-4 transverse process level provides effective postoperative analgesia for total hip arthroplasty. J Clin Anesth. 2018;44:68

19. Hong B, Bang S, Chung W, Yoo S, Chung J, Kim S. Multimodal analgesia with multiple intermittent doses of erector spinae plane block through a catheter after total mastectomy: a retrospective observational study. Korean J Pain. 2019; 32(3): 206214.

20. Bang S, Chung K, Chung J, Yoo S, Baek S, Lee S. The erector spinae plane block for effective analgesia after lung lobectomy: Three cases report. Medicine (Baltimore). 2019; 98(29): e16262.

21. Tulgar S, Senturk O. Ultrasound guided low thoracic erector spinae plane block for postoperative analgesia in radical retropubic prostatectomy, a new indication. J Clin Anesth. 2018;47:4-5. 